\title{
CORTICOTROPIN RELEASING HORMONE IS INVOLVED IN EXERCISE-INDUCED ELEVATION IN CORE TEMPERATURE
}

\author{
Pamela Johnson Rowsey ${ }^{1}$ and Matthew J. Kluger ${ }^{2}$ \\ 'Division of Kinesiology and ${ }^{2}$ Department of Physiology, University of Michigan, Ann Arbor, \\ Michigan, U.S.A.
}

(Accepted 6 August 1993)

\begin{abstract}
SUMMARY
To determine the involvement of corticotropin releasing factor (CRF) in exercise-induced elevation in core temperature of female rats, CRF antibody or vehicle was injected intracerebroventricularly (ICV) into rats that had free access to exercise wheels for 6 weeks. On the day of injection, there were no differences in body temperature or activity following these injections. However, exercising animals had a significantly attenuated daytime temperature when compared to vehicle control animals at one day postinjection with the CRF antibody. Although these animals have significantly lower body temperature than the animals that received vehicle, the injection of CRF antibody had no effect on locomotor activity. Therefore, this decrease in temperature is not due to a reduction in activity. These results suggest that $\mathrm{CRF}$ is involved in the exercise-induced elevation in daytime body temperature. Since this antibody also attenuates fevers caused by IL- $1 \beta$ (and presumably other cytokines), it is possible that the daytime elevation in body temperature of exercising rats is mediated by IL- $1 \beta$ or other cytokines.
\end{abstract}

\section{INTRODUCTION}

EXERCISE IN RATS (Rowsey et al., 1993a, 1993b; Satinoff et al., 1991) and hamsters (Conn et al., 1990) results in a rise in body temperature during the nighttime, when rats and hamsters are exercising, as well as during the daytime, when the animals are resting. What causes this daytime elevation in temperature? Does exercise induce a mild fever (i.e., change in thermoregulatory set-point) or is the rise in body temperature merely muscle-generated heat? To begin to answer these questions, we tested the hypothesis that this daytime elevation in temperature is mediated by tumor necrosis factor (TNF) or prostaglandins. Experiments in which antiserum to TNF was injected IP into rats at a dose which had been shown to block the biological actions of rat TNF both in vivo and in vitro did not have any effect on daytime temperature elevation seen in exercising female rats (Rowsey et al., 1993a). To determine whether prostaglandins are involved in the daytime elevation in body temperature, sodium salicylate was injected into rats at a dose that had been shown to block fevers caused by an IP injection of lipopolysaccharide (LPS). This did not result in any suppression of this daytime elevation in body temperature

Address correspondence and reprint requests to: M. J. Kluger, The Lovelace Institutes, 2425 Ridgecrest Drive, SE, Albuquerque, NM 87108. 
(Rowsey et al., 1993b). We concluded that neither TNF nor prostaglandins were involved in the daytime elevation in body temperature.

There are some data supporting the hypothesis that corticotropin-releasing factor (CRF) is involved in elevating core temperature in rats (Morimoto et al., 1993; Strijbos et al., 1992). Strijbos et al. (1992) injected rats with CRF (4.7 $\mu \mathrm{g} \mathrm{ICV)}$ and measured colonic temperature 30 and $60 \mathrm{~min}$ after injection. Colonic temperature was significantly increased at both time points $\left(1.20 \pm 0.1^{\circ} \mathrm{C}\right)$. There are also data supporting the hypothesis that CRF is involved in stress-induced fever in male rats (Morimoto et al., 1993). Exposure of rats to a novel environment results in a rise in body temperature. The effects of CRF on stress-induced fever was tested by injecting saline or $\alpha$-helical CRF ( $\alpha$-CRF receptor antagonist) ICV in stressed and unstressed animals. Psychological stress, in this study, was induced by switching the cages of rats for $60 \mathrm{~min}$. Injection of $\alpha$-helical CRF significantly attenuated stress-induced fever and locomotor activity. In unstressed animals, $\alpha$ helical CRF had no effect on body temperature or locomotor activity. Morimoto et al. (1993) also injected unstressed rats with CRF-41 $(1 \mu \mathrm{g}$ and $10 \mu \mathrm{g}) \mathrm{ICV}$. Injection of CRF41 caused a significant elevation in body temperature in a dose-dependent manner for the first 20 min postinjection. Body temperature reached a peak at $15 \mathrm{~min}$ after injection, then gradually declined. Injection of CRF-41 also significantly increased locomotor activity in these same animals.

CRF has been shown to be involved in the pyrogenic actions of cytokines such as interleukin-1 $\beta$ (IL-1 $\beta$ ) and interleukin-6 (IL-6). Blocking the biological actions of CRF within the central nervous system of the rat by central injection of a CRF antagonist or a neutralizing antibody to CRF significantly attenuated the pyrogenic responses to central injections of IL-1 $\beta$ and IL-6 (Rothwell, 1989; Rothwell et al., 1991). Rothwell (1989) injected animals ICV with a CRF receptor antagonist ( $\alpha$-helical CRF) $10 \mathrm{~min}$ prior to IL$1 \beta(50 \mathrm{ng})$. Injection of this antagonist completely prevented the rise in body temperature caused by IL-1 $\beta$. Busbridge et al. (1989) also injected animals with the same CRF antagonist or a monoclonal antibody to CRF followed by ICV injection of IL- $1 \beta$. Both treatments significantly attenuated the pyrogenic responses to IL- $1 \beta$. Intracerebroventricular injection of human recombinant IL-6 also caused significant increases in colonic temperature in rats (Rothwell et al., 1991), and the pyrogenic response to this cytokine was also inhibited when animals were pretreated with CRF antagonist. Based on these results, they concluded that the pyrogenic actions of IL- $1 \beta$ and IL-6 were dependent on the central actions of CRF.

Exercise causes the release of a circulating factor that exhibits endogenous pyrogen activity when injected into rats (Cannon \& Kluger, 1983). The goal of this study was to test the hypothesis that endogenous CRF is involved in the exercise-induced elevation in core temperature. We chose female rats as our animal model because they will run more than their male counterparts and produce a clear separation in both daytime and nighttime temperatures when compared to sedentary controls.

\section{MATERIALS AND METHODS}

\section{Animal Procedures}

Twenty female, specific pathogen free, Sprague-Dawley-CD (Charles River Laboratories, Portage, Michigan, U.S.A.) rats weighing 180-190 g at the time of Minimitter implantation, (Minimitter Inc., Sun River, Oregon, U.S.A.) were housed individually in Nalgene running cages ("exercising animals") for approximately 6 weeks. Sedentary 
animals were also individually housed in Nalgene cages (Nalge Co., Rochester, New York, U.S.A.). Animals were housed in a temperature controlled chamber at $23^{\circ} \mathrm{C}$ where a 12-h light/dark cycle was maintained with lights on at $0600 \mathrm{~h}$. Rats were fed standard rodent chow (Purina 5001) and tap water ad lib.

\section{Biotelemetry}

Temperature and motor activity were monitored continuously using the Dataquest, III system (Minimitter, Inc., Sun River, OR). Battery-operated transmitters were implanted into the peritoneal cavity of each animal. Animals were permitted 7 days of recovery before they were placed on running wheels or used for experiments involving injection of antibody to CRF. Each transmitter (model VMFH) was calibrated prior to implantation and at the end of the experiment. Each transmitter output frequency $(\mathrm{Hz})$ was received by an antenna placed under the floor of each cage and transferred to an IBM-PC. A change in position of the transmitter was recorded as an activity count. This biotelemetry system allows data to be monitored at 5-15 min intervals without disturbing the animal.

\section{Running Wheels}

The Nalge Company in conjunction with the Minimitter Company designed a cage that allows physiological monitoring by telemetry from animals housed in running wheel cages. Without disturbing the animals, this system allows easy, convenient measurement of the animals' physical activity in response to chemical or environmental stimuli. A fully assembled cage with wheel (Minimitter, Inc., Sun River, OR) is $(\mathrm{L} \times \mathrm{W} \times \mathrm{H})$ : 50 $\times 26.8 \times 36.4 \mathrm{~cm}$. Wheel revolutions are recorded when a magnetic switch has been engaged.

\section{Antibody to Corticotropin-Releasing Hormone}

The rat monoclonal antibody to CRF, PFU $83(62 \mathrm{mmol})$, was a gift from Dr. Frank Berkenbosch (Free University, Amsterdam). This antibody binds synthetic rCRF and blocks the ACTH-releasing effects of rCRF in vivo and in vitro (Van Oers \& Tilders, 1991). Central injection of rat monoclonal antibody to CRF has been shown to significantly attenuate the fever response to IL-1 $\beta$ (Busbridge et al., 1989; Rothwell, 1989).

\section{Interleukin-1 $\beta$}

The recombinant murine interleukin- $1 \beta(\mathrm{mIL}-1 \beta)$ was a gift from Dr. Ivan Otterness (Pfizer Corporation, Groton, Connecticut, U.S.A.).

\section{Surgery}

After 3 weeks of running, rats were implanted with ICV cannulae (Plastics One Product Co., Roanoke, Virginia, U.S.A.) and Minimitters. Animals were anesthetized with a mixture of Ketamine $(87 \mathrm{mg} / \mathrm{kg})$ and Xylazine $(13 \mathrm{mg} / \mathrm{kg})$ injected IM. First, a battery operated telemetry transmitter (Minimitter, Inc., Sunriver, OR) was implanted into the abdominal cavity for continuous monitoring of body temperature and activity, and then a $5 \mathrm{~mm}$ long ICV cannulae was implanted stereotaxically (Kopf's Instruments) into the lateral cerebral ventricle. The coordinates were $1 \mathrm{~mm}$ posterior to bregma and $0.5 \mathrm{~mm}$ lateral of the midline. Two small stainless steel screws were placed around the cannula and then dental acrylic was added around the cannula and screws. Then the guide cannulae was closed with a dummy cannula. When the dummy cannulae was tightly screwed in, it extended from the tip of the guide cannulae by no more than $0.2 \mathrm{~mm}$. At 
the time of injection, the animal was removed from its home cage, the dummy cannula was unscrewed, and the injection cannula with \#20 Polyethelene (PE) tubing was inserted into the cannula. The fluid was injected over $30 \mathrm{~s}$ using a Hamilton syringe attached to the PE tubing. During this time, the rat was held loosely with the head stabilized by one finger holding it just above the eyes. To minimize the increase in temperature due to the stress of handling, the rats were injected on two separate occassions with artificial cerebral spinal fluid (aCSF) not less than $24 \mathrm{~h}$ prior to the experiment. After injections, animals were returned to their home cages. At the end of the experiment, each rat was killed, its brain removed, and the location of the tip of the cannula was verified.

\section{Experimental Design}

The stock monoclonal antibody to CRF $(62 \mathrm{mmol})$ was reconstituted with $500 \mathrm{ml}$ of distilled water to bring the solution to $124 \times 10^{6} \mathrm{pmol} / \mathrm{ml}$. The stock solution was diluted 4700 -fold with aCSF. This was injected ICV in a volume of $5 \mu$ lo yield a dose of 131.9 pmol per rat, a dose of antibody similar to that shown to attenuate cytokine-induced fevers (Busbridge et al., 1989). The first experiment involved injecting the CRF antibody or vehicle ICV into sedentary rats. This was done to confirm, as in studies from other laboratories (Busbridge et al., 1989; Rothwell et al., 1989), that CRF does not have any effect on normal body temperature of female rats. The second experiment served as a positive control. Busbridge et al. (1989) showed that ICV injection of $130 \mathrm{pmol}$ of the CRF antibody into male rats led to the attenuation of IL- $1 \beta$-induced fever. This experiment was repeated in sedentary female rats. Lastly, to determine whether CRF had any role in exercise-induced changes in body temperature, a similar dose of the CRF antibody or vehicle, was injected ICV into female rats that had free access to exercise wheels for approximately 6 weeks.

\section{Statistical Analysis}

Data collected in 5-15 min intervals were collapsed into hourly averages for graphing and statistics. Data are presented as mean \pm SEM. All data were analyzed using Statview $512+$ (Abacus Concepts, Inc., Berkeley, CA). Student's $t$-tests were used to compare the changes in body temperatures of exercising rats with those of sedentary rats in response to the injections of CRF antibody. The alpha level was set at 0.05 level.

\section{RESULTS}

\section{CRF Antibody Has No Effect on Body Temperature of Sedentary Rats}

Figure 1 shows body temperature (Fig. 1A) and locomotor activity (Fig. 1B) of sedentary female rats injected ICV with antibody to CRF or vehicle. Injection of anti-CRF or vehicle occurred between 0830 and $0900 \mathrm{~h}$. Both groups of animals show a rise in temperature and activity due to handling from injection. However, there was no difference in body temperature or locomotor activity between rats injected with anti-CRF or with vehicle on the day of injection or 1 day postinjection.

\section{CRF Antibody Attenuates IL-I $\beta$-Induced Fever}

Figure 2 shows body temperature of sedentary female rats injected ICV with antiCRF or vehicle $10 \mathrm{~min}$ prior to ICV injection of mIL-1 $\beta(53 \mathrm{ng})$. Injection of anti-CRF or vehicle occurred between 0830 and $0900 \mathrm{~h}$. On the day of injection, both groups of animals increased their temperatures due to handling and stress of the injection and this 

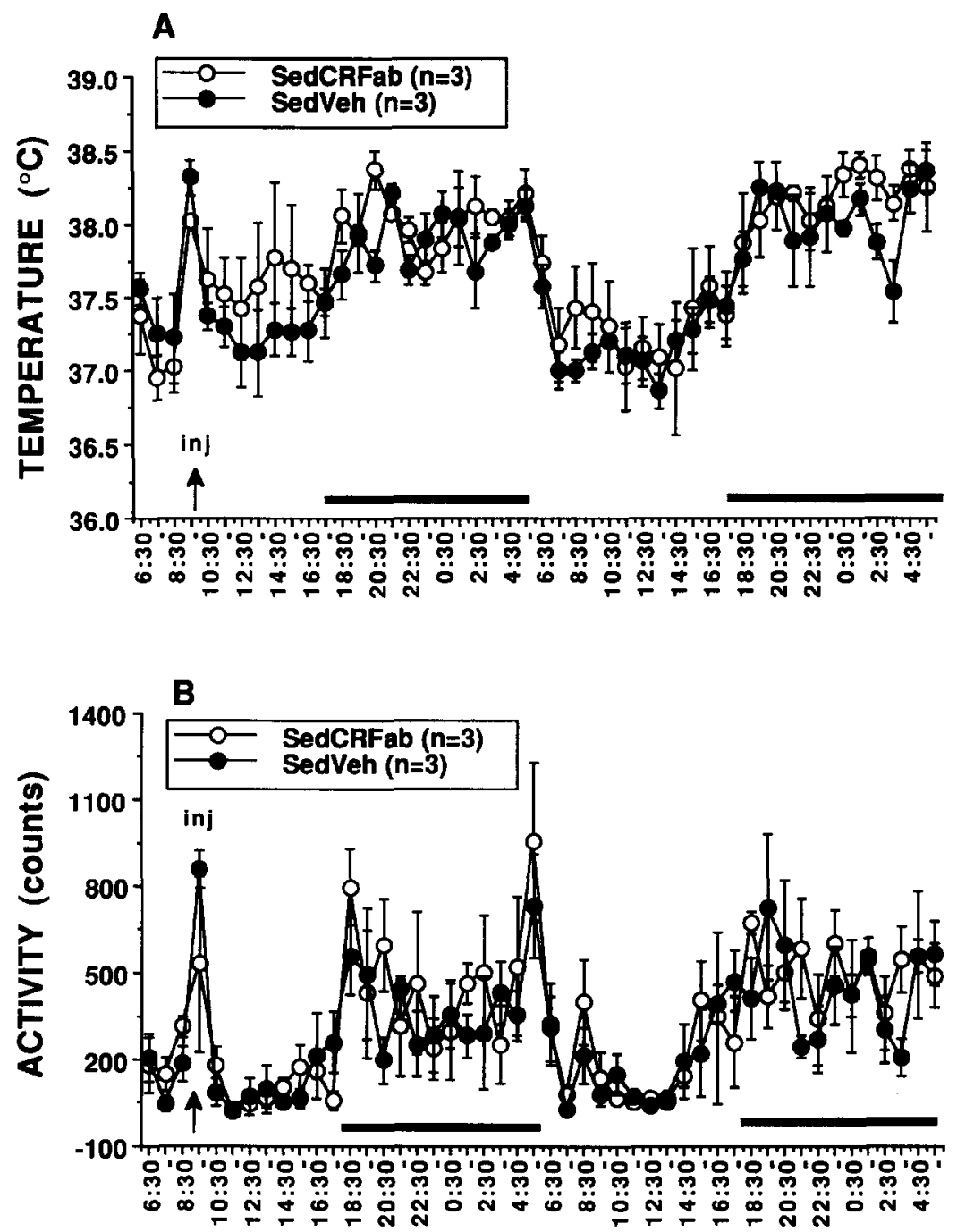

TIME (hours)

Fig. 1: Body temperature (A) and locomotor (nonwheel) activity (B) of sedentary female Sprague-Dawley rats injected with antibody to CRF or vehicle. Data represented as mean \pm SEM and are shown for 2 days. The injections were made at $0830-0900 \mathrm{~h}$ on the first day. Horizontal bar represents lights-off portion of the photoperiod.

effect subsided by $1030 \mathrm{~h}$. Injection of vehicle and IL-1 $\beta$ led to a fever that averaged $0.45^{\circ} \mathrm{C}$ higher $(39.63 \pm 0.07$ vs. $39.18 \pm 0.02)$ than that of rats injected with anti-CRF and IL-1 $\beta$ between 1200 and $1400 \mathrm{~h}(p=.02$ ) (Fig. 2). On the day prior to injection, average temperatures of both groups of animals between 1200 and $1400 \mathrm{~h}$ were almost identical (CRFab+IL-1 $\beta$ : $37.18 \pm 0.07^{\circ} \mathrm{C}$ vs. Vehicle $+\mathrm{IL}-1 \beta: 37.10 \pm 0.07^{\circ} \mathrm{C}$ ).

\section{Effect of CRF Antibody on Body Temperatures of Exercising Female Rats}

In previous studies from our laboratory (Rowsey et al., 1993a, 1993b) and in this study, we have found female Sprague-Dawley rats with access to exercise wheels 


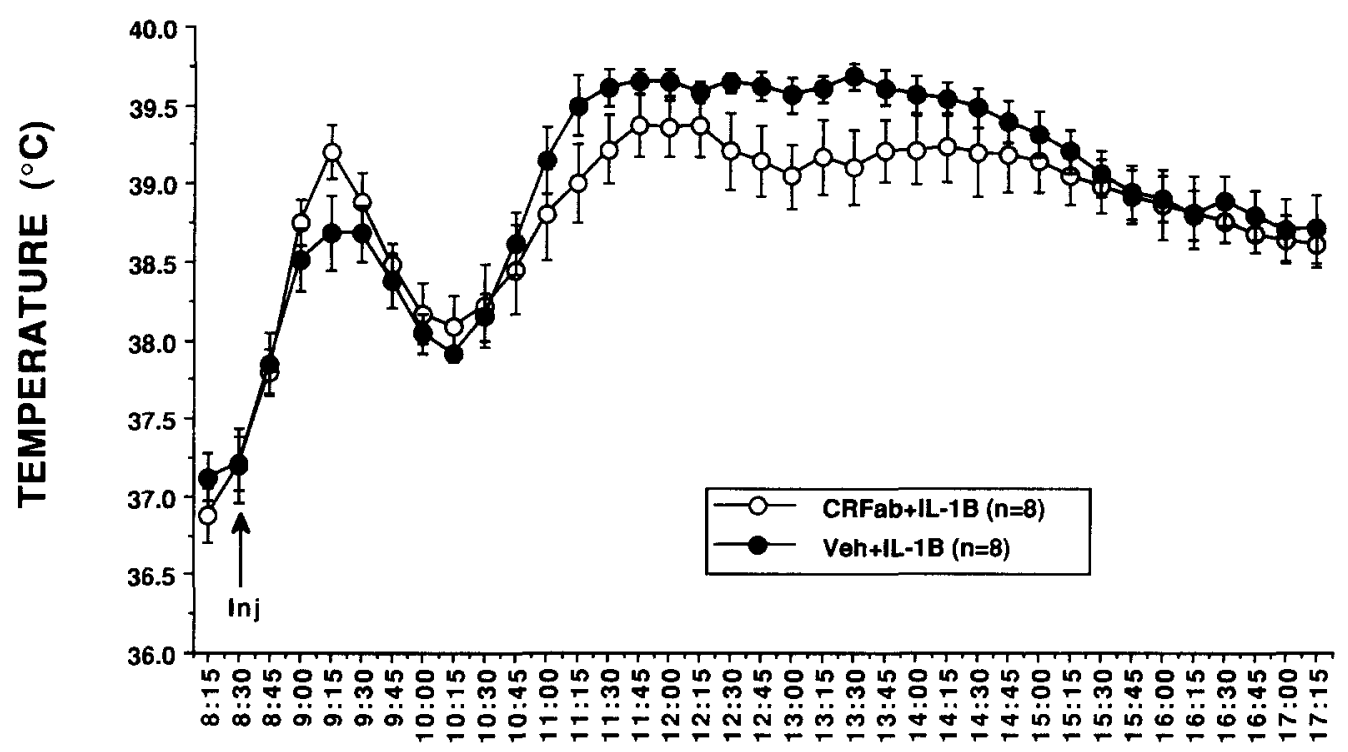

TIME (minutes)

FIG. 2: Body temperature of sedentary female Sprague-Dawley rats injected intracerebroventricularly with anti-CRF or Vehicle $10 \mathrm{~min}$ prior to mIL-1 $\beta(53 \mathrm{ng})$. Data represented as mean \pm SEM.

had an elevated body temperature, compared to rats with no access to exercise wheels (i.e., "sedentary") both during the period of voluntary exercise (nighttime) about $0.5^{\circ} \mathrm{C}(p=.0001)$ and the nonexercise period (daytime) about $0.3^{\circ} \mathrm{C}(p=.006)$. Figure 3 shows body temperature (Fig. 3A) and locomotor (nonwheel) activity (Fig. 3B) on the day of injection and one day following injection for exercising animals injected with either anti-CRF or vehicle. Animals were injected with anti-CRF or vehicle between 0830 and $0900 \mathrm{~h}$. Injection of CRF antibody or vehicle initially caused a rise in both temperature and locomotor activity in the exercising animals, but by $1 \mathrm{~h}$ postinjection the locomotor activity had returned to preinjection values. There were no differences in body temperature or activity during the night following these injections. The day after the ICV injections, the runners that received CRF antibody had a significantly attenuated body temperature between 0830 and $1530 \mathrm{~h}$ compared to runners that received vehicle (Fig. 3A) (RunCRFab: $37.23 \pm 0.06$ vs. RunVehicle: $37.57 \pm 0.14, p=.028$, Student's $t$-test) with the greatest significance in temperature occurring between 1200 and 1600h (RunCRFab: $37.19 \pm 0.05$ vs. RunVehicle: 37.61 $\pm 0.16, p=.011$, Student's $t$-test). Although these animals have significantly lower body temperature than the animals that received vehicle, the injection of CRF antibody had no effect on locomotor (nonwheel) activity (Fig. 3B) or on wheel running (Fig. 4), and thus, this decrease in temperature is not due to a decrease in locomotor activity. Although there is a slight reduction in body temperature and activity in the group of rats injected with CRF antibody during the second night following injection, these values were not significantly different from those of rats injected with vehicle.

Figure 4 shows the locomotor (wheel running) activity for animals that received 

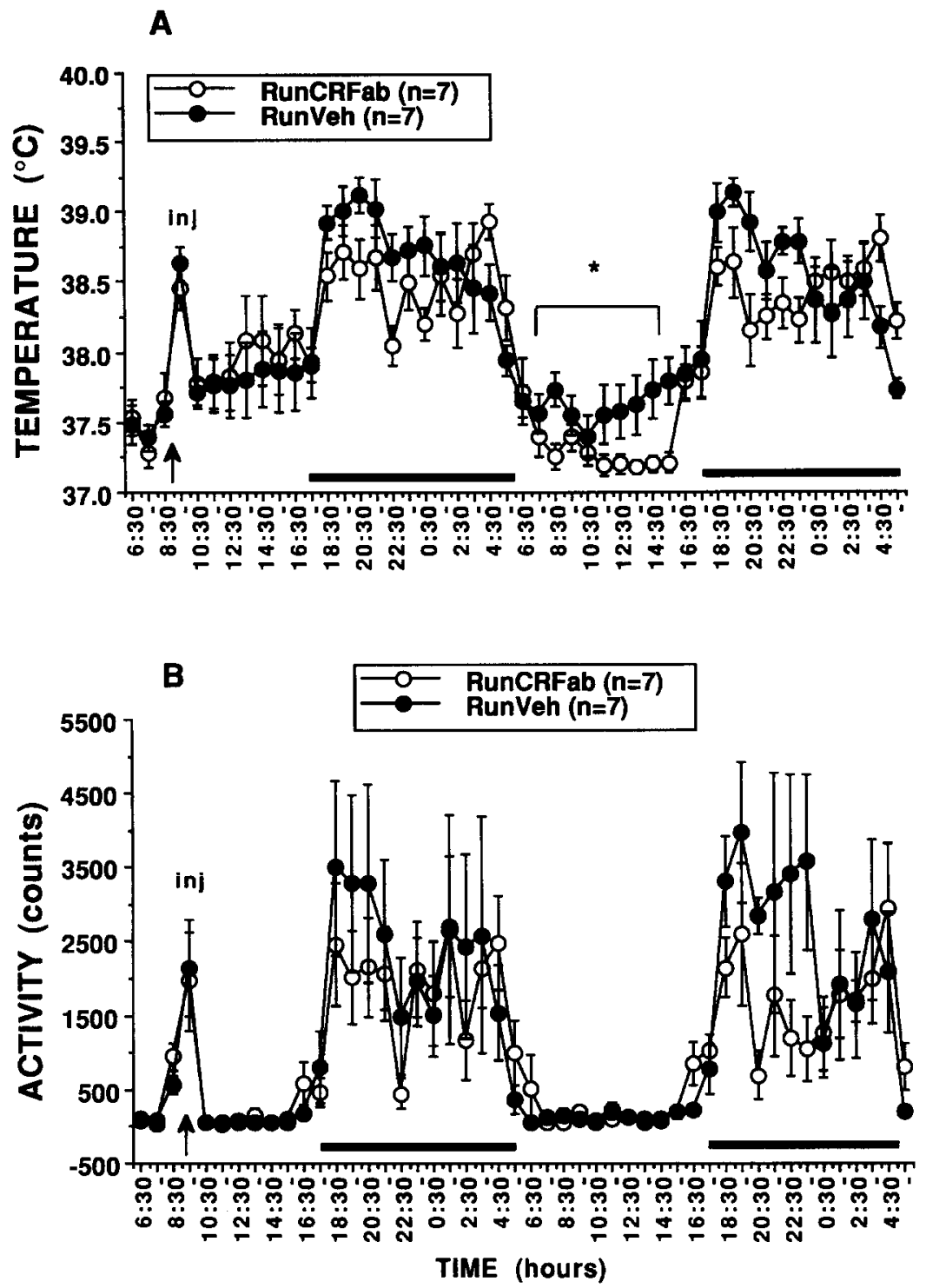

FIG. 3: Body temperature (A) and locomotor (nonwheel) activity (B) on the day of injection and one day following injection for exercising female Sprague-Dawley rats that received either antiCRF or Vehicle. Data represented as mean \pm SEM and are shown for 2 days. The injections were made at $0830-0900 \mathrm{~h}$ on the first day. Horizontal bar represents lights-off portion of the photoperiod.

anti-CRF or vehicle during the lights-on portion of the photoperiod (0815-1715h). One animal from the CRF antibody group was omitted from data analysis because of a computer error in collecting the data for this time period. On the day of injection, both groups of animals increased their running, when compared to preinjection day. During this time period, the animals that received anti-CRF tended to run more than the vehicle injected group, but this was not statistically significant $(p=.28)$. One 


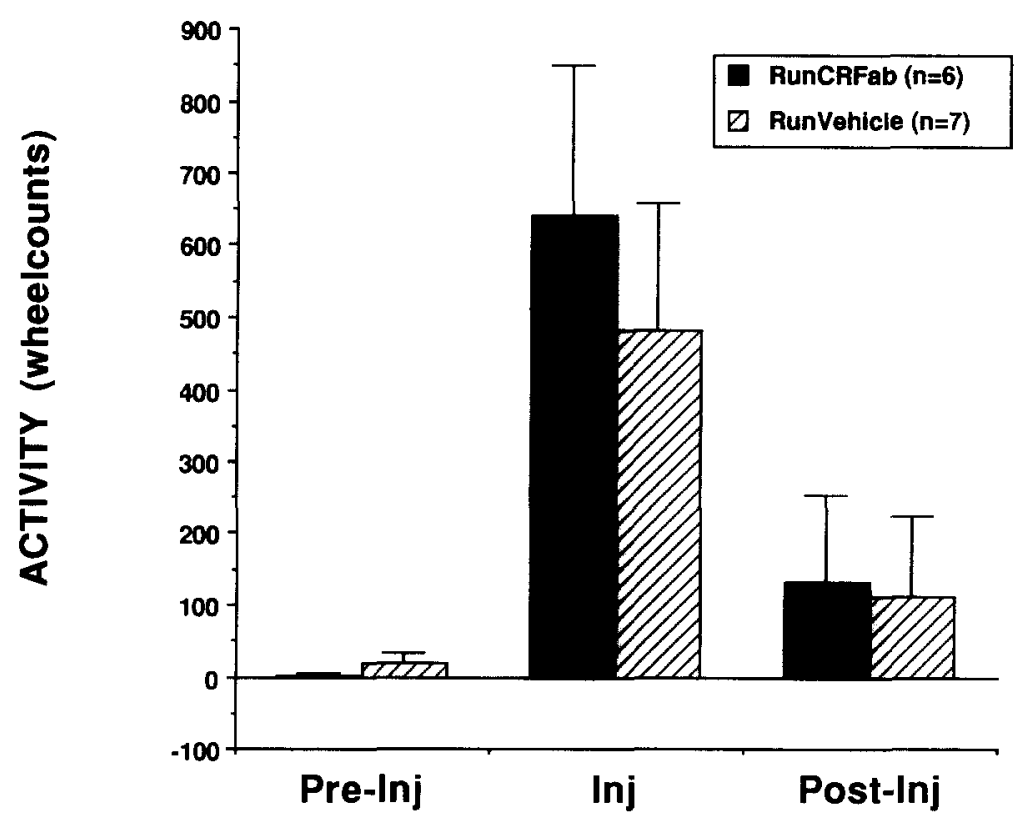

Fig. 4: Locomotor (wheel running) activity of exercising female Sprague-Dawley rats during the lights-on portion of the photoperiod $(0815-1715 \mathrm{~h})$ preinjection, the day of injection and the day following injection.

day postinjection, both groups of animals decreased their running when compared to injection day values.

\section{DISCUSSION}

These results support the hypothesis that endogenously produced CRF plays a role in the exercise-induced elevation in daytime body temperature in the female rat. AntiCRF had no effect on body temperature or locomotor activity of sedentary animals, yet did lower the daytime temperature of exercised rats compared to other exercised rats receiving vehicle. This effect on daytime temperature of exercised rats occurred the day after the injection and lasted for about $8 \mathrm{~h}$ of the lights-on portion of the photoperiod. We do not know why the effect of anti-CRF on body temperature was delayed until the day following the ICV injection.

Several studies have demonstrated that central nervous system CRF is involved in IL-1 $\beta$ fevers (Busbridge et al., 1989; Rothwell, 1989). Busbridge et al. (1989) pretreated male rats with a CRF antagonist or monoclonal antibody to CRF before ICV injection of IL-1 $\beta$. Injection of CRF antagonist almost completely prevented IL- $1 \beta$ fever response in male rats (94\% attenuation of fever). However, injection of monoclonal antibody significantly attenuated IL- $1 \beta$ responses in male rats but was less effective (36\% attenuation of fever) than the CRF antagonist (Busbridge et al., 1989). Rothwell (1989) also pretreated male rats with CRF antagonist or monoclonal antibody prior to ICV injection of IL- $1 \beta$ and reported a $100 \%$ attenuation of IL- $1 \beta$ fever when animals were pretreated with CRF antagonist. Data were not shown for CRF antibody's effect on body temperature in male rats, but it was reported that the CRF antibody inhibited, but did not prevent, 
the stimulatory effects of IL-1 $\beta$ (Rothwell, 1989). In our study, we used female rats (rather than male rats) and collected data via biotelemetry (rather than via rectal probe), which allows data to be collected over time without disturbing the animal. Injection of the anti-CRF antibody prior to IL- $\beta$ decreased the fever in our animals by $21 \%$. Our data are qualitatively similar to those of Busbridge et al. (1989) and Rothwell's (1989), providing further evidence of CRF's involvement in IL-1 $\beta$-induced fever.

Exercise generally leads to an elevated core temperature, which is often accompanied by a release of cytokines into the blood. During infection-induced fever, it is believed that cytokines such as IL-1 or IL-6, trigger the elevation in the thermoregulatory setpoint by increasing arachidonic acid metabolites, primarily prostaglandins of the $\mathrm{E}$ series (PGE). However, data presented from a previous study (Rowsey et al., 1993b) lead us to conclude that while the rise in the daytime temperature in rats that voluntarily exercise at night may be the result of an elevated thermoregulatory set-point, this elevation in temperature is not reversed by injection of sodium salicylate, and therefore does not appear to be mediated by PGE. It is apparent that if this exercise-induced elevation in core temperature is indeed a change in thermoregulatory set-point, that this change in set-point is not occurring through a traditional cyclooxygenase pathway. These results suggest that CRF plays a role in exercise-induced elevated body temperature possibly through the pyrogenic actions of cytokines such as IL- $1 \beta$ or IL- 6 , which have been shown to cause fever via CRF. Based on our data, and those of others, we speculate that some cytokine, perhaps IL- $1 \beta$, working via CRF is involved in this exercise-induced elevation of core temperature.

Acknowledgments: This work was supported in part by NIH Grant AI 27556. Partial support for Pamela Johnson Rowsey was from a Grant from the Horace H. Rackham Graduate School. We thank (posthumously) Dr. Frank Berkenbosch for providing us with the antibody to CRF, and Dr. Ivan Otterness for providing us with the murine IL- $1 \beta$.

\section{REFERENCES}

Busbridge NJ, Dascombe MJ, Tilders FJH, van Oers JWAM, Linton EA, Rothwell NJ (1989) Central activation of thermogenesis and fever by interleukin- $1 \beta$ and interleukin- $\alpha$ involves different mechanisms. Biochem Biophys Res Commun 162:591-596.

Cannon JG, Kluger MJ (1983) Endogenous pyrogen activity in human plasma after exercise. Science 220:617-619.

Conn CA, Borer KT, Kluger MJ (1990) Body temperature rhythm and response to pyrogen in exercising and sedentary hamsters. Med Sci Sports Exerc 22(5):636-642.

Morimoto A, Nakamori T, Morimoto K, Tan N, Murakami N (1993) The central role of corticotropin-releasing factor (CRF-41) in psychological stress in rats. J Physiol 460:221-229.

Rothwell NJ (1989) CRF is involved in the pyrogenic and thermogenic effects of interleukin- $1 \beta$ in the rat. Am J Physiol 256(19):E111-E115.

Rothwell NJ, Busbridge NJ, LeFeuvre RA, Hardwick AJ, Gauldie J, Hopkins SJ (1991) Interleukin6 is a centrally acting endogenous pyrogen in the rat. Can J Physiol Pharmacol 69:1465-1469.

Rowsey PJ, Borer KT, Kluger MJ (1993a) Tumor necrosis factor is not involved in exerciseinduced elevation in core temperature. Am J Physiol 265:R1351-R1354.

Rowsey PJ, Borer KT, Kluger MJ (1993b) Role of prostaglandins in exercise-induced core temperature elevation in female Sprague-Dawley rats. Am J Physiol 265:R1121-R1125.

Satinoff E, Kent S, Hurd M (1991) Elevated body temperature in female rats after exercise. Med Sci Sports Exerc 23(11):1250-1253.

Strijbos PJ, Hardwick AJ, Relton JK, Carey F, Rothwell NJ (1992) Inhibition of central actions of cytokines on fever and thermogenesis by lipocortin-1 involves CRF. Am J Physiol 263:E632-E636.

Van Oers, JWAM, Tilders, FJH (1991) Antibodies in passive immunization studies: Characteristics and consequences. Endocrinology 128(1):496-503. 